\title{
Activation Volume of Secondary Relaxation
}

\section{Soheil Sharifi}

Department of Physics, University of Sistan and Baluchestan, Zahedan, Iran. Email: sharifi@phys.usb.ac.ir, soheil.sharifi@gmail.com

Received January $30^{\text {th }}, 2011$; revised March $28^{\text {th }}, 2011$; accepted April $2^{\text {nd }}, 2011$.

\begin{abstract}
Glass forming materials are characterized by a complex relaxation pattern, which evolves over several time decades. Dielectric spectroscopy has proven particularly useful for studying such scenario as it is able to monitor the dielectric dynamics of a system over a range up to 16 time decades. In this work we study effect of thermodynamic history on activation volume of secondary relaxation inside the glassy forming systems, Poly [(phenyl glycidyl ether)-co-formaldehyde], (PPGE) and 1,18-bis ( $p$ methoxyphenyl) cyclohexane (BMPC), with two different type of secondary relaxation. Our results show that at bout systems, activation volume of secondary depends to the thermodynamic history.
\end{abstract}

Keywords: Activation Volume, Secondary Relaxation, Glassy State, Pressure Effects

\section{Introduction}

Secondary relaxations are the only dynamic processes active on a measurable time scale in the glassy state. Its importance is two folds: usually the secondary relaxation is the main mechanism of glasses for dissipating external stresses, [1-4]. Moreover, from the dynamic point of view secondary relaxation is a suitable easily accessible source of information about the glassy state. Despite this importance, the investigation about secondary relaxation has been usually limited to the description of its temperature and pressure dependences, but scarce attention has been devoted to other characterizations, [5-11]. For example, the secondary process is usually investigated in the glassy state, which is a non equilibrium state of materials, but not too much is known about its dependence on the history of formation of the glass, $[12,13]$. The consequence of the non equilibrium nature of glasses on the thermodynamic and structural properties has been largely studied, and interpretations in terms of phenomenological models have been proposed. Recently thermodynamic history dependence was noted in some secondary processes, as for example, different relaxation frequencies of secondary processes are observed consequently to cooling with different rates, or after verifications combining different sequences of cooling and compression steps. The reason why secondary processes are influenced by the thermodynamic history of the glass is not clear at all, and the microscopic mechanisms at the basis of such dependence are unknown. The situation is even more complicated since the molecular mechanisms at the basis of secondary process are not clear at all. A greater sensitivity to the thermodynamic history is expected for secondary processes of intermolecular origin, Johari-Goldstein (JG) but experimental data lack. In this work, we present experimental studies varying pressure and temperature pressures of secondary processes in several glass formers. Also, we will present the study of the influence of thermodynamic history on the secondary process of different molecular glass formers, namely Poly[(phenyl glycidyl ether)-co-formaldehyde], (PPGE), 1,18-bis ( $p$ methoxyphenyl) cyclohexane (BMPC).

\section{Experimental}

\subsection{Materials}

Poly[(phenyl glycidyl ether)-co-formaldehyde] (PPGE), with average molecular weight, M.W. $=345 \mathrm{~g} / \mathrm{mol}$, and $T_{g}$ around $258 \pm 1 \mathrm{~K},[10]$, were supplied by Aldrich Chemicals. and 1,18-bis ( $p$ methoxyphenyl) cyclohexane (BMPC), with average molecular weight, M.W. $=296 \mathrm{gr} / \mathrm{mol}$, and $T_{g}$ at ambient pressure around $246 \pm 1 \mathrm{~K}$, was synthesised in the laboratory of professor H. Sillescu and obtained from Prof. M. Paluch. Glassy 1,18-bis ( $p$ methoxyphenyl) cyclohexane (BMPC) show two secondary relaxations whose molecular origin is not clear at all, $[14,15]$.

\subsection{Methods}

Dielectric measurements were carried out by a dielectric spectrometer (Alpha-Novocontrol) in the frequency interval $10^{-2}-10^{7} \mathrm{~Hz}$. For measurements at ambient pres- 
sure the sample was placed in a parallel plate cell (diameter $30 \mathrm{~mm}$, gap $0.1 \mathrm{~mm}$ ) and the temperature control was performed with a precision better than $0.1 \mathrm{~K}$ by using a dry-nitrogen stream based system. For measurements at high pressure the sample was placed in a parallel plate cell (diameter $20 \mathrm{~mm}$, gap $0.05 \mathrm{~mm}$ ) that, properly insulated from the external environment, was located inside a pressure chamber. Pressure variations $(0.1-600 \mathrm{MPa})$ were generated by a manual pump and transmitted to the sample through silicon oil. A liquid circulator connected to a jacket, wrapped around the pressure room, allowed the control of temperature (353 $233 \mathrm{~K}$ ) within $0.1 \mathrm{~K}$.

\section{Results}

Representative isothermal dielectric loss spectra of the investigated glass formers in the glassy state are presented in Figure 1. We acquired dielectric spectra by varying pressure from $0.1 \mathrm{MPa}$ up to the maximum value of $600 \mathrm{MPa}$. For all the systems we can observe a peak, corresponding to the secondary relaxation: In the case of PPGE, we observe at high frequencies a rise in the signal partly due to the presence of a faster relaxation, which however is never completely visible with our apparatus for high pressure measurements. In the case of 1,18-bis ( $p$ methoxyphenyl) cyclohexane (BMPC) at low frequencies the tail of the structural peak is still present in some spectra. The $\beta$-relaxation in Poly[(phenyl glycidyl ether)-co-formaldehyde],(PPGE), moves to lower frequencies faster than the secondary relaxation in 1,18-bis ( $p$ methoxyphenyl) cyclohexane (BMPC), Figure 1. It is reported that the $\beta$-secondary process of Poly[(phenyl glycidyl ether)-co-formaldehyde] (PPGE) is of the Johari-Goldstein (JG) type [12], whereas the $\beta$-process of 1,18 -bis ( $p$ methoxyphenyl) cyclohexane (BMPC) is not Johari-Goldstein (Non-JG), [13].

In this work, we studying the secondary relaxation in glassy materials prepared along the thermodynamic paths represented in Figure 2 and Table 1. In particular we focused on the $\beta$-process of 1,18-bis ( $p$ methoxyphenyl) cyclohexane (BMPC) and Poly[(phenyl glycidyl ether)-co-formaldehyde] (PPGE). Along the path (A), we vitrify the material by isothermal compression (to the point B) and finally we cooled the material to the temperature represented by the point $\mathrm{D}$. Subsequently to this procedure the secondary relaxation was studied during isothermal decompression (D-C). Along path (B), we vitrified the system by cooling at ambient pressure (to the point $\mathrm{C}$ having the same value of $\mathrm{T}$ of the point $\mathrm{D}$ ) and then we investigated the secondary relaxation along the isothermal path with increase pressure.

Figure 3 shows maxima frequency as a function of pressure for 1,18-bis ( $p$ methoxyphenyl) cyclohexane

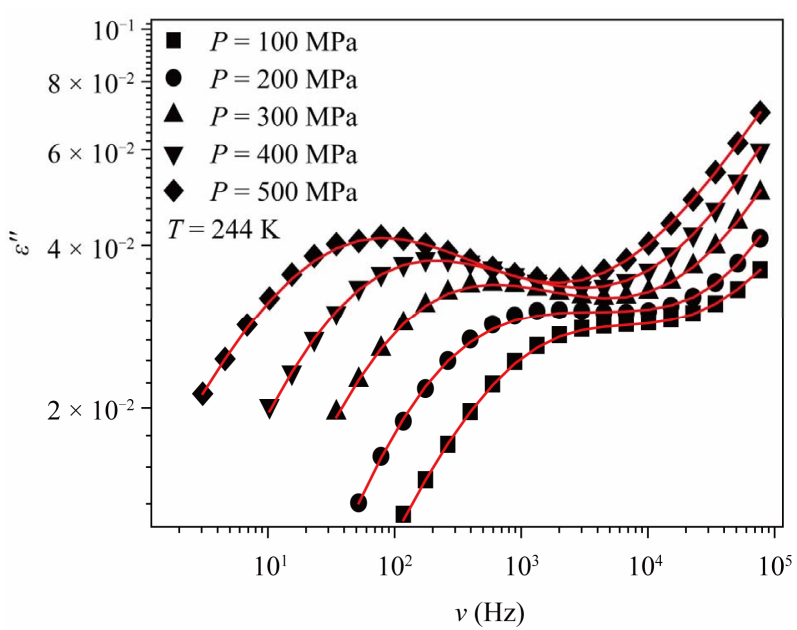

(a)

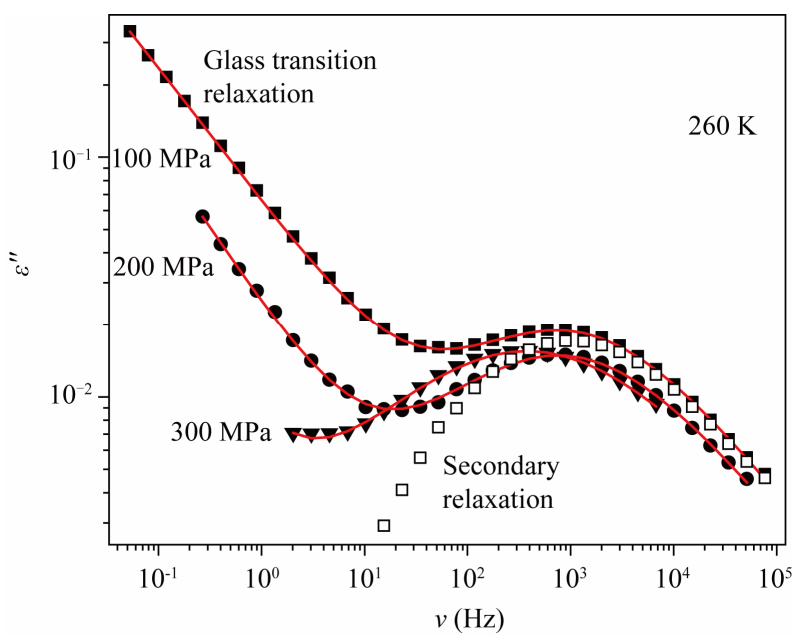

(b)

Figure 1. (a) Isothermal loss spectra of Poly[(phenyl glycidyl ether)-co-formaldehyde], (PPGE) at different pressure, The points are results of experiment and lines are the fit of Equation (1); (b) Isothermal loss spectra of 1,18-bis ( $p$ methoxyphenyl) cyclohexane (BMPC) at various pressures in the glassy state. Secondary peak slightly shifts with pressure towards lower frequencies. Empty squares represents the loss spectrum measure at 100 MPa corrected for the contribution of the $\alpha$-peak. The points are results of experiment and lines are the fit of Equation (1).

Table 1. Parameters of the thermodynamic paths represented in Figure 2.

\begin{tabular}{cccccc}
\hline & \multicolumn{3}{c}{ Path A } & \multicolumn{2}{c}{ Path B } \\
\cline { 2 - 6 } & $T_{i}, P_{i}$ & $T_{f}, P_{f}$ & $T_{g}(p)$ & $T_{i}, P_{i}$ & $T_{f}, P_{f}$ \\
\hline \multirow{2}{*}{ BMPC } & $260 \mathrm{~K}$ & $234 \mathrm{~K}$ & $260 \mathrm{~K}$ & $260 \mathrm{~K}$ & $234 \mathrm{~K}$ \\
& $0.1 \mathrm{MPa}$ & $600 \mathrm{MPa}$ & $150 \mathrm{MPa}$ & $0.1 \mathrm{MPa}$ & $600 \mathrm{MPa}$ \\
& & & & & \\
PPGE & $283 \mathrm{~K}$ & $253 \mathrm{~K}$ & $283 \mathrm{~K}$ & $342 \mathrm{~K}$ & $253 \mathrm{~K}$ \\
& $0.1 \mathrm{MPa}$ & $450 \mathrm{MPa}$ & $200 \mathrm{MPa}$ & $0.1 \mathrm{MPa}$ & $450 \mathrm{MPa}$
\end{tabular}




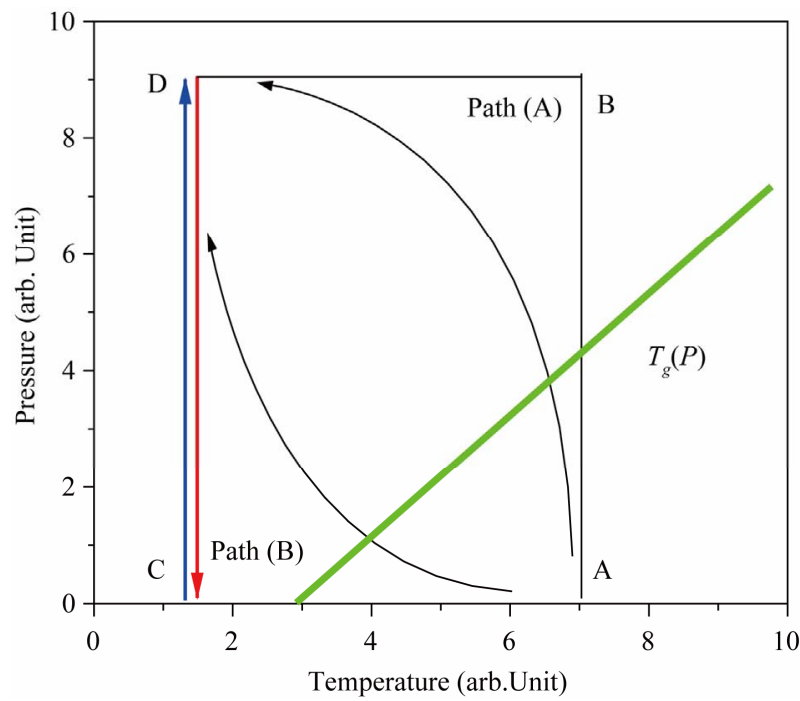

Figure 2. Schematic representation of the thermodynamic paths for study the thermodynamic history we consider two path that path $(\mathrm{A})$ is the material by isothermal compression to the point $B$ and cooled to lower temperature, The path (B), liquid is cooled at ambient pressure to the glassy state and then pressure increased.

(BMPC). We analyzed spectra in terms of a $\mathrm{HN}$ function for the structural process, Equation (1) and a Cole-Cole equation ( $\beta=1$ at Equation (1)) for the secondary relaxation.

$$
\varepsilon(\omega)=\varepsilon_{\infty}+\frac{\Delta \varepsilon}{\left[1+\left(i \omega \tau_{\beta}\right)^{1-\alpha}\right]^{\beta}}
$$

In the case of Poly[(phenyl glycidyl ether)-co-formaldehyde], (PPGE), we added a HN equation for fitting the faster secondary relaxation ( $\gamma$-relaxation). The shape parameters used in the $\mathrm{HN}$ equation for the $\gamma$-relaxation in these systems were obtained from fitting of spectra at very low temperature and ambient pressure where the $\gamma$-relaxation can be clearly observed. Moreover, a similar procedure was used for fitting the tail of the structural peak when present in the spectra. The pressure dependence of secondary relaxation for 1,18-bis ( $p$ methoxyphenyl) cyclohexane (BMPC) and Poly[(phenyl glycidyl ether)-co-formaldehyde], (PPGE) with different origin is represented in Figure 3 for 1,18-bis ( $p$ methoxyphenyl) cyclohexane (BMPC) and Figure 4 for PPGE. At the 1,18-bis ( $p$ methoxyphenyl) cyclohexane (BMPC), the glass formed along path (A) is less sensitive to change in pressure than that formed along path (B). In analogy, we saw the same effect in the PPGE Figures 3 and $\mathbf{4}$. We calculate activation volume from Equation (2), which is reported in Table 2.

$$
v_{\text {max }}^{\beta}(P)=v_{P=0} \exp \left[-P \Delta V_{\beta} /\left(K_{b} T\right)\right]
$$

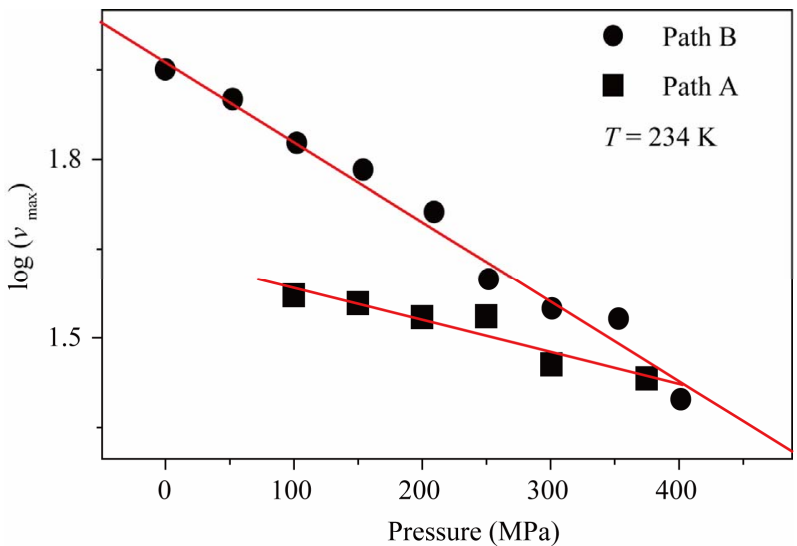

Figure 3. $\log \left(v_{\max }\right)$ as a function of pressure for 1,18-bis ( $p$ methoxyphenyl) cyclohexane (BMPC) with increase of pressure (circle points-Path (B)) and decrease pressure (square points-Path $(\mathrm{A}))$.

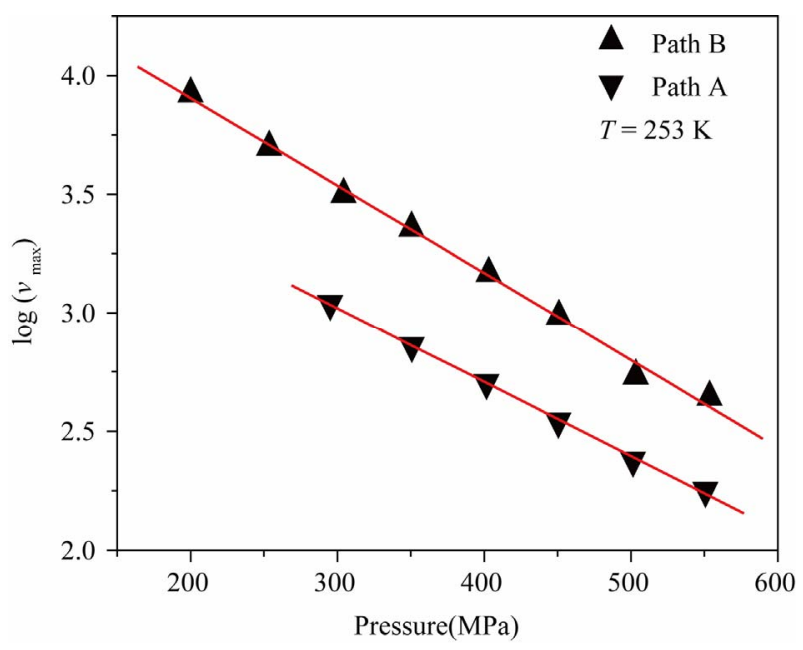

Figure 4. $\log \left(v_{\max }\right)$ as a function of pressure for Poly[(phenyl glycidyl ether)-co-formaldehyde], (PPGE) with increase of pressure (up triangle points) and decrease pressure (down triangle points).

Table 2. Activation volume of secondary relaxation in Poly[(phenyl glycidyl ether)-co-formaldehyde], (PPGE) and 1,18-bis ( $p$ methoxyphenyl) cyclohexane (BMPC) with decrease and increase pressure and thermodynamic paths of Table 1.

\begin{tabular}{cccc}
\hline & $T(K)$ & $\begin{array}{c}\Delta V_{\beta}(\mathrm{ml} / \mathrm{mol}) \\
\text { Increase Pressure }\end{array}$ & $\begin{array}{c}\Delta V_{\beta}(\mathrm{ml} / \mathrm{mol}) \\
\text { Decrease Pressure }\end{array}$ \\
\hline BMPC & 234 & $5.2 \pm 0.1$ & $2.6 \pm 0.1$ \\
PPGE & 253 & $17.9 \pm 0.5$ & $15.2 \pm 0.2$ \\
\hline
\end{tabular}

where $v_{P}=0$ is the relaxation frequency at ambient pressure and temperature corresponding to that of the isotherm of investigation, $\Delta V_{\beta}$ is the activation volume of the secondary $(\beta$-) process. 


\section{Discussions}

Density variations affect the secondary relaxation process in the investigated systems. In fact, the relaxation frequency decreases on increasing pressure at fixed temperature, which correspond to an increase of density (Figure 1). The extent of the density effect on the secondary dynamics depends on the investigated system. Accordingly, secondary relaxation in Poly[(phenyl glycidyl ether)-co-formaldehyde], (PPGE) is more sensitive to density (larger values for $\Delta V$ ) than 1,18-bis ( $p$ methoxyphenyl) cyclohexane (BMPC) (smaller values for $\Delta V$ ) (Table 2). Systems vitrified along path A are compressed in the liquid state and then in the glassy state, whereas systems vitrified along the path B are compressed only in the glassy state (Figure 2). We can expect that glasses prepared along path A have larger density than those prepared along path B. In fact, compressing is usually more effective in increasing the density than cooling and the compressibility of the liquid is larger than that of the glass. This should be reason of the different of the behavior of secondary relaxation when we increase pressure or decrease pressure that we can see it on the behaviors of activation volume of secondary relaxation inside the glassy state.

\section{Conclusions}

In this study, we performed several experiments with the aim of characterizing the dependence of the secondary relaxations on the thermodynamic history used to produce the glass. We observed a relation between the dependence on thermodynamic history of the activation volume and compressibility. In particular, using different thermodynamic histories we observed in 1,18-bis ( $p$ methoxyphenyl) cyclohexane (BMPC) and Poly[(phenyl glycidyl ether)-co-formaldehyde], (PPGE), that the $\beta$ process has larger activation volumes (larger pressure sensitivity) in glasses prepared with lower densities. This characteristic is independent from the microscopic origin of the process, as in Poly[(phenyl glycidyl ether)-coformaldehyde], (PPGE), it is of the Johari-Goldstein (JG) type and in 1,18-bis ( $p$ methoxyphenyl) cyclohexane (BMPC) is non- Johari-Goldstein (Non-JG).

\section{Acknowledgements}

This work was supported by the University of Sistan and Baluchestan.

\section{REFERENCES}

[1] K. L. Ngai and M. Paluch, "Classification of Secondary Relaxation in Glass-Formers Based on Dynamic Properties," Journal of Chemical Physics, Vol. 120, No. 1, 2004, pp. 857-873. doi:10.1063/1.1630295
[2] M. Vogel, P. Medick and E. A. Rossler, "Secondary Relaxation Processes in Molecular Glasses Studied by Nuclear Magnetic Resonance," Annual Reports on NMR Spectroscopy, Vol. 56, 2005. pp. 231-299. doi:10.1016/S0066-4103(05)56005-8

[3] L. Leuzzi, "Static and Dynamic Glass-Glass Transitions: A Mean-Field Study," Philosophical Magazine, Vol. 88, No. 33, November 2008, pp. 4015-4023.

[4] M. Zuriaga, L. C. Pardo, P. Lunkenheimer, J. L. Tamarit, N. Veglio, M. Barrio, F. J. Bermejo and A. Loidl, "New Microscopic Mechanism for Secondary Relaxation in Glasses," Physical Review Letters, Vol. 103, No. 7, August 2009, pp. 1-4. doi:10.1103/PhysRevLett.103.075701

[5] D. Prevosto, S. Capaccioli, M. Lucchesi, P. A. Rolla and K. L. Ngai, "Does the Entropy and Volume Dependence of the Structural $\alpha$-Relaxation Originate from the Johari-Goldstein $\beta$-Relaxation?" Journal of Non-Crystalline Solids, Vol. 355, No. 10-12, 2009, pp. 705-711. doi:10.1016/j.jnoncrysol.2008.09.043

[6] J. D. Stevenson and P. G. Wolynes, "A Universal Origin for Secondary Relaxations in Supercooled Liquids and Structural Glasses," Nature Physics, Vol. 6, November 2009, pp. 62-68.

[7] R. Casalini and C. M. Roland, "Anomalous Properties of the Local Dynamics in Polymer Glasses," Journal of Chemical Physics, Vol. 131, No. 11, September 2009, pp. 1-7. doi:10.1063/1.3223279

[8] R. Casalini and C. M. Roland, "Effect of Crosslinking on the Secondary Relaxation in Polyvinylethylene," Journal of Polymer Science Part B: Polymer Physics, Vol. 48, No. 5, 2010, pp. 582-587. doi:10.1002/polb.21925

[9] K. Kessairi, S. Capaccioli, D. Prevosto, M. Lucchesi, S. Sharifi and P. A. Rolla, "Interdependence of Primary and Johari-Goldstein Secondary Relaxations in Glass-Forming Systems," The Journal of Physical Chemistry B, Vol. 112, March 2008, pp. 4470-4473.

[10] D. Prevosto, S. Capaccioli, S. Sharifi, K. Kessairi, M. Lucchesi and P. A. Rolla, "Secondary Dynamics in Glass Formers: Relation with the Structural Dynamics and the Glass Transition," Journal of Non-Crystalline Solids, Vol. 353, No. 47-51, December 2007, pp. 4278-4282.

[11] K. Kessairi, S. Capaccioli, D. Prevosto, M. Lucchesi and P. A. Rolla, "Relaxation Dynamics in Tert-Butylpyridine/ Tristyrene Mixture Investigated by Broadband Dielectric Spectroscopy," Journal of Chemical Physics, Vol. 127, No. 17, November 2007, pp. 1-11. doi:10.1063/1.2784190

[12] S. Sharifi, D. Prevosto, S. Capaccioli, M. Lucchesi and M. Paluch, "Effect of Thermodynamic History on Secondary Relaxation in the Glassy State," Journal of Non-crystalline Solids, Vol. 353, No. 47-51, December 2007, pp. 4313-4317.

[13] D. Prevosto, S. Sharifi, S. Capaccioli, P. A. Rolla, S. Hensel-Bielowka and M. Paluch, "New Experimental Evidence about Secondary Processes in Phenylphthalein-Dimethylether and 1,1'-bis(p-Methoxyphenyl)Cyclohexane," Journal of Chemical Physics, Vol. 127, No. 11, 
September 2007, pp. 1-6. doi:10.1063/1.2771169

[14] R. Casalini and C. M. Roland, "Scaling of the Supercooled Dynamics and Its Relation to the Pressure Dependences of the Dynamic Crossover and the Fragility of Glass Formers," Physical Review B, Vol. 71, No. 1, January 2005, pp. 1-11.
[15] R. Casalini, M. Paluch and C. M. Roland, "The Dynamics Crossover Region in Phenol- and Cresol-Phthalein-Dimethylethers under Different Conditions of Pressure and Temperature," Journal of Physics: Condensed Matter, Vol. 15, No. 11, March 2003, pp. S859-S867.

doi:10.1088/0953-8984/15/11/310 